\title{
Situação nutricional e alimentar de pré-escolares no semi-árido da Bahia (Brasil): I.Avaliação antropométrica*
}

\author{
Nutritional status of pre-school children of the semi-arid region of Bahia \\ (Brazil): I. Anthropometric status
}

\author{
Leonor M.P. Santos, Ana Marlúcia O. Assis, Celia M. Baqueiro, Gilca M.C. \\ Quaglia, Saul S. Morris e Mauricio L. Barreto
}

Escola de Nutrição. Universidade Federal da Bahia. Salvador - Brasil (L.M.P.S., A.M.O.A., C.M.B. e G.M.C.Q.), Maternal and Child Epidemiology Unit, Department of Epidemiology and Population Sciences. London School of Hygiene and Tropical Medicine (S.S.M.), Instituto de Saúde Coletiva, Universidade Federal da Bahia. Salvador - Brasil (M.L.B.)

\begin{abstract}
Foram estudados 754 pré-escolares de áreas urbanas de sete municipios do semi-árido da Bahia, Brasil. Os objetivos foram determinar a prevalência de déficit ponderal e estatural, indicativos de desnutrição atual e/ou pregressa e sua associaçáa com a idade, sexo, renda em salário-mínimo (SM), escolaridade materna e adequaçăo do consumo alimentar. Encontrou-se $22,9 \%$ de crianças com altura/idade abaixo de $-2,0$ DP (desnutrição pregressa), 19,1\% com peso/idade e 3,6\% com peso/altura abaixo de $-2,0$ DP (desnutrição atual). Em relaçáo ao inquérito dietético somente $6,8 \%$ das crianças haviam consumido no dia anterior uma dieta que suprisse os requerimentos energéticos para sua faixa etária. Houve forte associação entre os indicadores $\mathrm{A} / \mathrm{l}$ e $P / l$ inadequados com renda familiar per capita ( $p=0,001$ e $p=0,000$, respectivamente); crianças de famílias com renda per capita < 1/4 SM tinham duas vezes mais chance de estar desnutridas se comparadas com as do estrato de renda $\geq 1 / 2 \mathrm{SM}$. Em relação ao P/A como também $P / \mid$, os pré-escolares estudados no semi-árido apresentaram prevalèncias significativamente superiores às encontradas por pesquisa nacional realizada na mesma época ( $p=0,047$ e $p=0,000$, respectivamente). Esses achados surpreendem, já que nas últimas décadas tem sido demonstrada no Brasil uma melhorla slgnifivatlva na desnutriçăo e mortalidade infantil e parecem indicar que as crianças do semi-arido não lograram ainda alcançar os mesmos beneficios que o restante da populaçâo infantil brasiteira.
\end{abstract}

Anemia, epidemiologia. Hemoglobinas, análise.

\section{Introdução}

A Bahia é o maior Estado do Nordeste e um dos que apresentam maior diversidade tanto ecológica como social e econômica entre as suas unidades naturais; contudo, em que pese a importância desse Estado, inexistem dados de base populacional sobre a situação alimentar e nutricional de sua populaçāo. Estado ímpar no Brasil tanto pela composiçāo étnica como pela riqueza cultural de sua gente, a Bahia é marcada pelas injustas diferenças da sociedade brasileira: em um extremo encontra-se a tecnologia de ponta empregada em seu moderno Pólo Petroquímico e na prospecção e exploração do petróleo; no outro a mais retrógrada estrutura fundiária e a mais atrasada tecnologia agrícola utilizada em grande parte do semi-árido, responsáveis pelos desastres sociais previsíveis que acompanham as secas periódicas. Apesar das condiçőes climáticas adversas e mesmo considerando a acentuada migração em direçāo aos grandes centros urbanos existem, de 
acordo com o censo de 1991, 5.915.000 habitantes nos 257 municípios do semi-árido da Bahia. A área incluída no polígono das secas na Bahia corresponde a $393.000 \mathrm{~km}^{2}$, ou seja, mais que $2 / 3 \mathrm{da}$ área total do Estado ${ }^{5.19}$ (Figura1).

Condiçōes de privação crônica em populações como essas sāo responsáveis por atrasos no desenvolvimento pondero-estatural de crianças e adolescentes que podem ser detectados através de antropometria; indicadores antropométricos têm sido extensivamente usados como "proxy" para o estado nutricional da população. Alguns levantamentos no Estado da Bahia têm demonstrado alta prevalência de inadequação nos indicadores antropométricos em pré-escolares. Estudos realizados na década dos 80's em zonas rurais e urbanas de Sapeaçu (regiåo do recôncavo), Barreiras (regiāo do serrado) e Cansançāo (região semi-árida) têm demonstrado alta prevalência de déficits no peso para a idade ${ }^{1.7 .8}$. Destes 3 estudos publicados, apenas o último localizou-se na região semi-árida do Estado.

Duas pesquisas de saúde e nutrição de âmbito nacional, realizadas na década de 80 , tiveram um plano amostral que nāo permitiu analisar os dados antropométricos em nível estadual, mas somente para as grandes regiôes brasileiras. Além disto tanto a Pesquisa Nacional sobre Saúde Materno Infantil e Planejamento Familiar (PNSMIPF), realizada em 1986 como parte da Pesquisa Demográfica e de Saúde (DHS) ${ }^{3}$, como a Pesquisa Nacional de Saúde e Nutrição (PNSN) realizada em 1989 pelo Instituto Nacional de Alimentação e Nutrição (INAN)' nāo levantaram dados sobre carências de micronutrientes ou sobre o consumo alimentar.

Em relação ao consumo alimentar, indicador de grande importância para o conhecimento da cadeia causal da desnutrição, os estudos são ainda mais escassos, talvez pela dificuldade de obtenção de dados confiáveis através do inquérito dietético, por mais criteriosa que seja sua aplicação. Em nível nacional os únicos dados que se dispōe são do Estudo Nacional de Despesa Familiar (ENDEF) realizado entre 1974-75, com amostras representativas das grandes regióes brasileiras, onde foi avaliado por sete dias consecutivos o consumo alimentar da famnlia por pesagem direta. Os dados de consumo alimentar, analisados por unidade familiar, foram parcialmente publicados em $1982^{6}$ e não permitem avaliar o consumo individual das crianças.

Este estudo teve como objetivo caracterizar a situação alimentar e nutricional da população de pré-escolares do semi-árido da Bahia, sobretudo no que diz respeito ao desenvolvimento ponderoestatural, hipovitaminose $\mathrm{A}$ e anemia. Este primeiro relato descreve aspectos da pesquisa e apresenta os resultados de antropometria e de consumo energético e protéico; dados de anemia e hipovitaminose A encontram-se em outros trabathos nessa série de publicações ${ }^{218}$.

\section{Material e Método}

\section{Delimitação do Semi-Árido}

Optou-se por adotar a delimitação do semiárido baiano utilizada pela SUDENE (Superintendência de Desenvolvimento do Nordeste) que inclui 257 municípios da Bahia ${ }^{19}$.

A definição de Unidade Natural do Sertão da SEPLANTEC (Secretaria de Planejamento, Ciência e Tecnologia da Bahia), que adota como indicadores a vegetação de caatinga e o clima semiárido, ou seja, índice de aridez superior a 30, foi útil na seleção final da área do estudo.

\section{Seleção da Área}

As duas regióes mais áridas da Bahia (índice de aridez superior a 50) localizam-se ao Norte, incluindo o Município de Paulo Afonso, e em uma área central do Estado, circunvizinha ao Município de

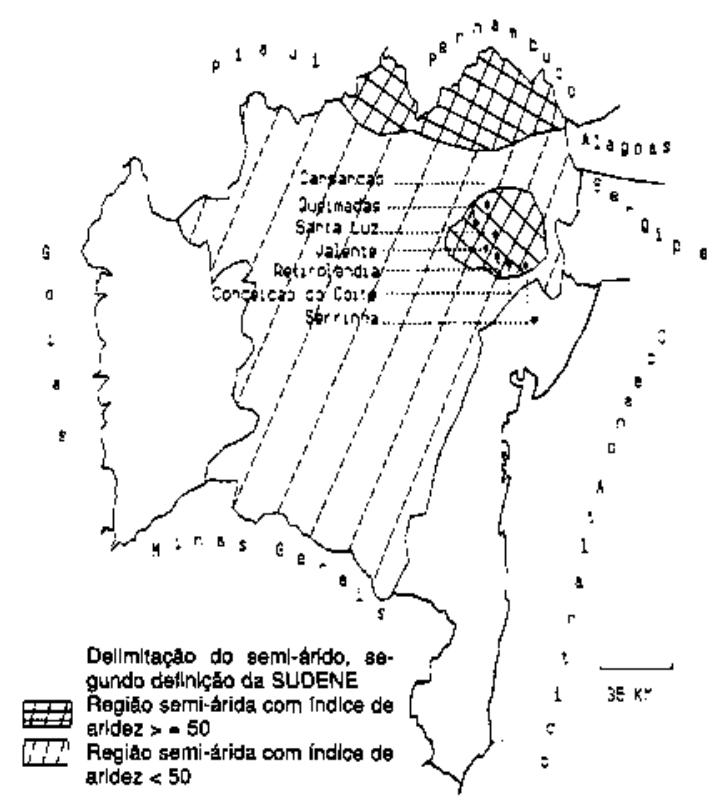

Figura 1 - Localização das 7 cidades estudadas no semiárido do Estado da Bahia. 
Queimadas. Ao norte do Estado existem áreas extensas em processo de desertificação, sendo portanto uma região esparsamente habitada; a população da regiāo é largamente concentrada na zona urbana do Município de Paulo Afonso (população

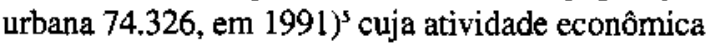
gira em torno da Usina Hidroelétrica que abastece quase toda a região Nordeste. Portanto, este município nāo pode ser considerado como típico do semiárido baiano. Decidiu-se, assim, localizar o estudo na regiāo central (Figura 1), que apresenta grande semelhança socioeconômica e cultural com o restante do sertāo nordestino, incluindo as áreas urbanas de Cansançāo, Queimadas, Santa Luz, Valente, Conceição do Coité, Retirolândia e Serrinha (populações urbanas de $6.900,8.451$, $12.695,6.410,19.993,3.860$ e 37.799 , respectivamente, em 1991).

\section{Seleção da Amostra}

A seleção da amostra foi feita pelo levantamento casa a casa de todas as famílias com crianças de zero a seis anos, residentes nos bairros periféricos carentes dos municípios (os bairros foram escolhidos segundo a informação das lideranças locais). No caso de famílias com mais de uma criança nesta faixa etária, procedia-se ao sorteio de uma criança por família. Foram selecionadas 910 crianças, sendo que em 156 casos (17\% da amostra) os pais nāo compareceram ao exame clínico e antropométrico e/ou não consentiram na coleta de sangue, resultando em uma amostra de 754 crianças.

\section{Coleta de Dados e Equipe de Campo}

A equipe básica para coleta de dados foi composta por nutricionistas, médico, técnica em patologia clínica e auxiliares de pesquisa; toda a equipe foi treinada na aplicação do questionário socioeconômico, no inquérito dietético e na coleta de dados antropométricos. Duas auxiliares de enfermagem integravam a equipe nos dias de coleta de sangue. Os dados foram coletados entre janeiro e junho de 1989 pela mesma equipe, que após completar um município se deslocava para o próximo. Durante as visitas domiciliares foram coletados dados demográficos, sociais, econômicos e dieteticos; em seguida a família era convocada a levar a criança em jejum ao Posto de Saúde em data aprazada, quando eram realizados o exame clínico, as medidas antropométricas e a coleta de sangue.

\section{Inquérito Dietético}

O consumo alimentar de energia e proteína foi avaliado através de inquérito dietético recordatório de 24 h respondido pela mãe ou responsáve ${ }^{22}$. Foi possível obter informaçāo quantitativa sobre o consumo alimentar de 616 pré-escolares; as perdas amostrais foram assim distribuídas: 30 crianças (4\% da amostra) consumiam um volume não quantificável de leite materno; 46 crianças $(6 \%$ da amostra) faziam as refeiçōes principais fora do lar (creche); e em 62 casos ( $8 \%$ da amostra) a informação fornecida pela mãe ou responsável foi incompleta não permitindo a correta análise do inquérito.

\section{Antropometria}

Medidas antropométricas foram realizadas com um mínimo de vestimentas e sem sapatos. Utilizaram-se para o peso balanças Filizola Pediátrica (até 23 meses) e Filizola Antropométrica ( 24 meses ou mais). O comprimento (até 23 meses) e a estatura ( 24 meses ou mais) foram medidos com precisão de $0,5 \mathrm{~cm}$ em antropômetros de madeira construídos especialmente para tal firm. O peso foi obtido de 753 crianças enquanto a altura foi obtida de 742 casos (98\% da amostra). Empregou-se o padrão internacional para a comparaçāo dos dados antropométri$\cos (\mathrm{NCHS})^{16}$, o mesmo adotado pelo Ministério da Saúde na elaboraçāo do Cartão da Criança.

\section{Controle de Qualidade e Processamento de Dados}

A consistência dos dados coletados nos questionários era verificada por outro entrevistador e pelos supervisores; nos poucos casos em que se verificaram inconsistências, nova coleta de dados era feita nos domićlios imediatamente, antes da equipe se deslocar da cidade. Os dados foram digitados em microcomputador utilizando o programa DENTRY/SPSS-PC +, que além de organizar o banco de dados permite definir parâmetros para crítica, minimizando erros de digitação. Os dados antropométricos foram analisados com EPINUT, módulo do Epi Info. Análises quantitativas de consumo alimentar empregaram o Programa de Apoio à Decisão em Nutrição, versão $1^{*}$. Nas análises estatíticas foram utilizados os programas SPSSPC+ e Epi Info.

\footnotetext{
* Programa cedido pela Escola Paulista de Medicina
} 


\section{Análise de Dados}

Consideraram-se como inadequados os casos com peso/idade, altura/idade ou peso/altura abaixo de $-2,0$ desvios-padrāo escore (DP), conforme sugerido pela $\mathrm{OMS}^{20}$. Foi empregada a classificação de Gomez $z^{9}$ que considera com desnutrido de grau I a criança com adequaçāo $P / 1$ entre $90,0 \%$ e $75,1 \%$; como de grau II entre $75,0 \%$ e $60,1 \%$ e como grau III quando abaixo de $60,0 \%$ da adequação do peso para idade. Para o cálculo de adequação de consumo de proteína e energia (conceito mínimo) foram utilizadas as recomendaçōes da FAO/OMS ${ }^{4}$. No caso de proteína procedeu-se à correção para a qualidade da dieta considerando para a dieta brasileira um fator de digestibilidade de $82 \%$ e um escore de amino ácidos de $97 \%$ (dieta mista de arroz/feijão/milho) ${ }^{4}$. Para análise de renda domiciliar o valor da remuneraçāo de todos os membros foi totalizado e expresso em fração do salário-mínimo mensal vigente na época; renda per capita foi obtida dividindo pelo número de pessoas que residiam no domicílio. Alguns autores têm adotado o ponto de corte abaixo de $1 / 4$ do salário-mínimo per capita para definir famílias em nível de pobreza absoluta no Brasil ${ }^{13.1}$. Foram adotadas a prevalência e razão de prevalência (RP) como medidas de associaçāo. $O$ Qui quadrado de Pearson, o teste de tendência de Mantel Haenzel e a probabilidade exata de Fisher (quando apropriada) foram usados para testar a significância a nível de $95 \%$ de confiança.

\section{Devolução dos Dados à Comunidade}

Para fins de devolução de dados à comunidade, a adequação peso/idade foi calculada em campo, e este resultado, juntamente com o de hemoglobina ${ }^{2}$, foi entregue às mães. Primeiramente realizou-se uma discussão com a comunidade sobre as causas e a prevenção da desnutrição e anemia, seguida pela entrega dos resultados. Logo após houve atendimento individual pelas nutricionistas e pela médica da equipe a todas as crianças com desnutrição de graus II ou III e/ou anemia, que consistia na anamnese alimentar e na orientação dietética, além da prescrição de sulfato ferroso para os casos de anemia grave (hemoglobina abaixo de 9,5 g/di).

\section{Resultados}

\section{Características Demográficas}

Dos 754 pré-escolares estudados, 373 eram do sexo masculino $(49,5 \%)$ e 381 do sexo feminino $(50,5 \%)$, com idades variando de 1 a 72 meses. As famílias estudadas apresentaram uma mediana de 6 pessoas residindo no mesmo domicílio, variando de 2 a 17 indivíduos. Em relação ao número de crianças por família, a mediana foi de 2 para os préescolares (variação de 1 a 7) e de 1 para os escolares (variação de 0 a 8). Das famílias estudadas, $61,3 \%$ tinham mais de um pré-escolar; nestes casos integrou a amostra somente a criança índice (sorteada).

\section{Características Sociais e Econômicas}

Das famílias estudadas, $13 \%$ apresentavam chefe de sexo feminino; o trabalho fora do lar foi declarado por $31 \%$ das mães, sendo $58 \%$ dos casos em tempo integral e $42 \%$ em tempo parcial (6 horas ou menos por dia). A amostra estudada era proveniente de famílias de baixa renda: $1,3 \%$ das famílias declararam-se sem renda alguma; $28,4 \%$ das famílias recebiam menos de 1 saláriomínimo (SM) mensal e 72,9\% das familias menos de dois SM; nāo foi possível obter a informaçāo de rencia para 42 famnlias ( $6 \%$ da amostra). Para todas as análises subsequientes optou-se por empregar renda per capita em fraçāo de SM, obtida conforme detalhado na metodologia. Os resultados referentes à renda per capita e escolaridade materna estão apresentados na Tabela 1: mais da metade das famílias $(52,0 \%)$ recebiam menos de $1 / 4$ de SM per capita; $41,7 \%$ das māes de crianças estudadas declararam-se analfabetas e $16,7 \%$ sabiam ler mas nāo haviam frequientado o primeiro grau.

Dos domícilios pesquisados $65,9 \%$ tinham acesso a água encanada (intradomiciliar ou torneira externa), $11,0 \%$ tinham destino adequado para o esgoto (rede geral ou fossa séptica) e o destino do lixo doméstico era adequado em $24,1 \%$ deles (coletado, enterrado ou queimado) (Tabela 1).

\section{Antropometria}

A Figura 2 ilustra a distribuição de frequiência dos três indicadores antropométricos comparada com a distribuição na população de referência (NCHS); pode-se notar em todos os casos um desvio da curva para a esquerda, caracterizando para a população estudada alta prevalência de inadequação, em especial para os indicadores altura/idade e peso/idade. A prevalência dos indicadores altura/idade, peso/altura e peso/idade 
Tabela 1- Indicadores sociais, económicos e de saneamento básico nas familias e domicilios de pré-escolares no semi-árido da Bahia - 1989.

\begin{tabular}{llr}
\hline Renda familiar per capta & $<1 / 4 \mathrm{SM}$ & $52,0 \%$ \\
(N=712) & $1 / 2$ a $1 / 4 \mathrm{SM}$ & $34,0 \%$ \\
& $\geq 1 / 2 \mathrm{SM}$ & $14,0 \%$ \\
Escolaridade materna & Analfabeta & $41,7 \%$ \\
(N=744) & Alfabetizada & $16,7 \%$ \\
& Primária & $36,6 \%$ \\
& Secundária & $5,4 \%$ \\
Origem da água & Caminhäo/outra & $20,3 \%$ \\
(N=753) & Poço/rio & $13,8 \%$ \\
& Encanada & \\
& (interna/externa) & $65,9 \%$ \\
Esgotamento sanitário & Valaffossa rudimentar $89,0 \%$ \\
(N=752) & Fossa séptica & $2,7 \%$ \\
& Rede geral & $8,4 \%$ \\
Destino do lixo & Terreno/vala & $75,9 \%$ \\
(N=704) & Enterrado/queimado & $6,8 \%$ \\
& Coletado & $17,3 \%$ \\
\hline
\end{tabular}

abaixo de -2,0 DP encontra-se na Tabela 2, comparada com os resultados para a população urbana do Nordeste da pesquisa nacional realizada também em 1989 (PNSN)'s. Não houve evidência de que a prevalência do indicador altura/idade inadequado fosse diferente nos dois inquéritos $(p=0.668)$, mas em relação ao peso/idade e peso/altura as prevalências encontradas na população do semi-árido foram quase o dobro das encontradas na PNSN, sendo as diferenças estatisticamente significativas $(p=0.000$ e $p=0,047$, respectivamente).

$O$ indicador peso/idade foi também analisado por outros métodos de classificação. Pela classificação de Gomez encontrou-se $44.6 \%$ de desnutrição de grau I. $11,6 \%$ de grau II e $0,8 \%$ de grau III. Já em relação ao percentil. $22,3 \%$ das crianças encontravam-se abaixo do percentil 3 e $19,1 \%$

Tabela 2 - Comparaçäo da prevaléncia de indicadores antropométricos abaixo de $-2,0$ desvios-padrảo (DP) em crianças pré-escolares do semi-árido da Bahia - 1989 , com resultados para o Nordeste urbano da Pesquisa Nacional de Saúde e Nutriçāo (PNSN) - 1989.

\begin{tabular}{|c|c|c|c|}
\hline Pesquisa & $\begin{array}{l}\text { Altura/ } \\
\text { Idade }\end{array}$ & $\begin{array}{l}\text { Peso/ } \\
\text { Idade" }\end{array}$ & $\begin{array}{l}\text { Peso/ } \\
\text { Altura". }\end{array}$ \\
\hline $\begin{array}{l}\text { Semi-árido da Bahia } \\
\text { (presente estudo) } \\
(\mathrm{N}=742 \text { para } \mathrm{A} / \mathrm{l} \text { e } \mathrm{P} / \mathrm{A}) \\
(\mathrm{N}=753 \text { para } \mathrm{P} / \mathrm{l})\end{array}$ & $22,9 \%$ & $19,1 \%$ & $3,6 \%$ \\
\hline $\begin{array}{l}\text { PNSN Nordeste urbano } \\
\text { (Monteiro \& Cervini) } \\
(\mathrm{N}=850 \text { para } \mathrm{A} / / \text { e } \mathrm{P} / \mathrm{A}) \\
(\mathrm{N}=866 \text { para } \mathrm{P} / \mathrm{l})\end{array}$ & $23,9 \%$ & $10,3 \%$ & $2,0 \%$ \\
\hline
\end{tabular}

${ }^{*} p=0,648{ }^{* x} p=0,000^{* * *} p=0,047$

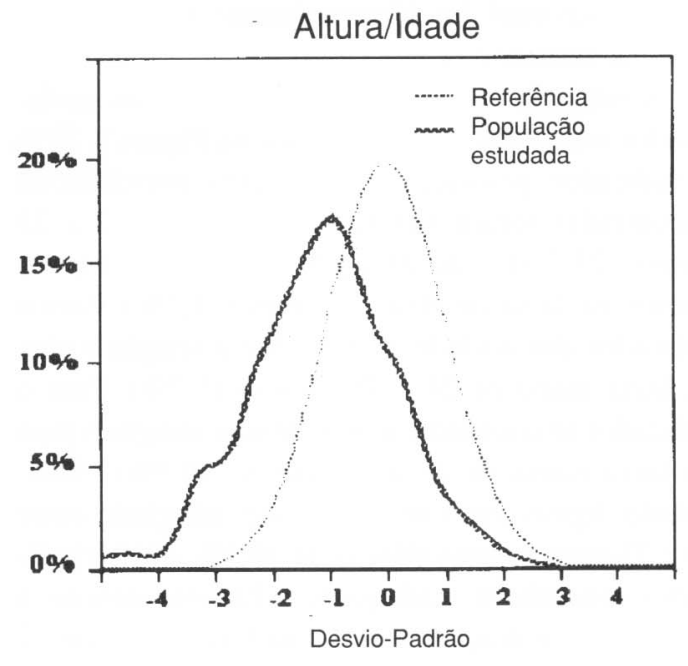

Peso/ldade

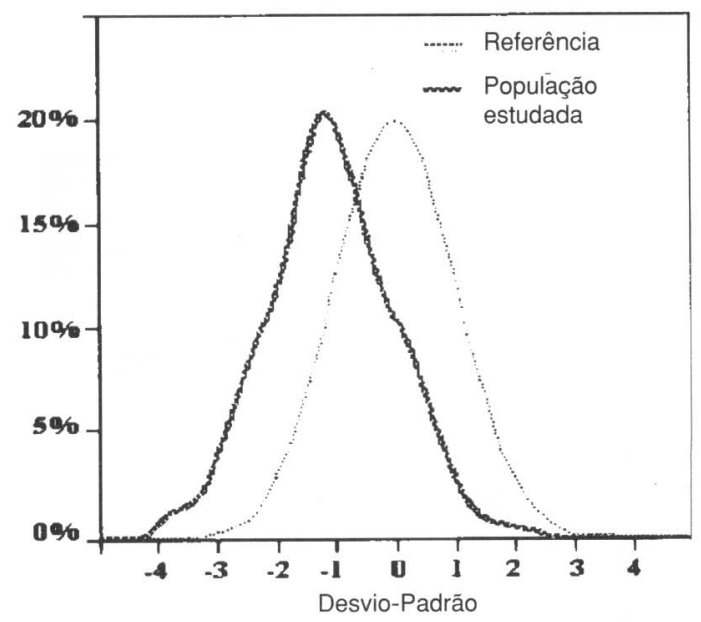

Peso/Altura

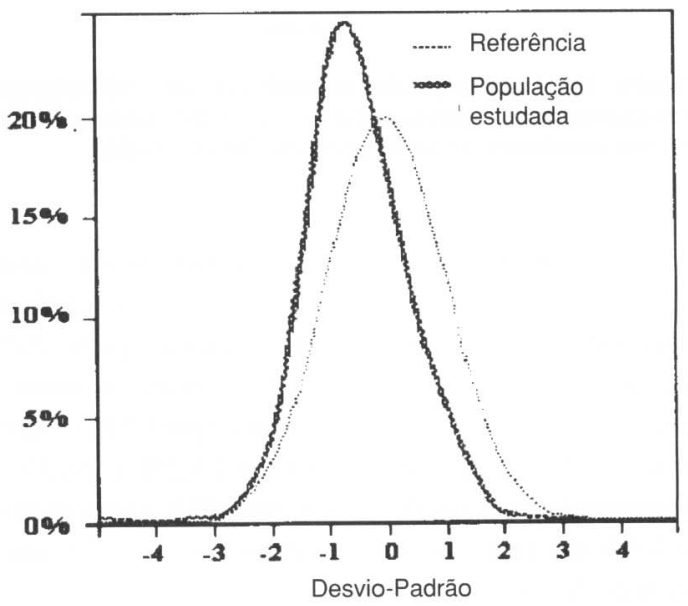

Flgura 2 - Distribuição dos indicadores antropométricos em crianças pré-escolares no semi-árido da Bahia - 1989. 
entre o percentil 3 e 10 , totalizando $41,4 \%$ consideradas desnutridas ${ }^{10}$.

A variação dos índices antropométricos inadeguados com a idade está ilustrada na Figura 3. Para o indicador peso/idade as maiores prevalências encontradas foram nas faixas etárias de 12 a 23 meses $(27,7 \%)$ e de 24 a 35 meses $(27,4 \%)$, e a menor, na faixa de 60 a 72 meses $(11,7 \%)$. Para o indicador altura/idade, a prevalência atingiu o pico na faixa etária de 24 a 35 meses $(11,7 \%)$. Para o indicador altura/idade, a prevalência atingiu o pico na faixa etária de 24 a 35 meses $(30,9 \%)$, diminuindo depois dos 3 anos de idade, atingindo entre 60 e 72 meses a prevalência de $18,2 \%$. Para o indicador peso/altura inadequado, chamou atenção a grande concentraçāo de casos na faixa etária de 12 a 23 meses, o que correspondeu a uma prevalência de $13,4 \%$; nas outras faixas etárias a prevalência de $P / A$ inadequado variou de $3,9 \%$ (menor que 12 meses) até $0,9 \%$ (de 60 a 72 meses).

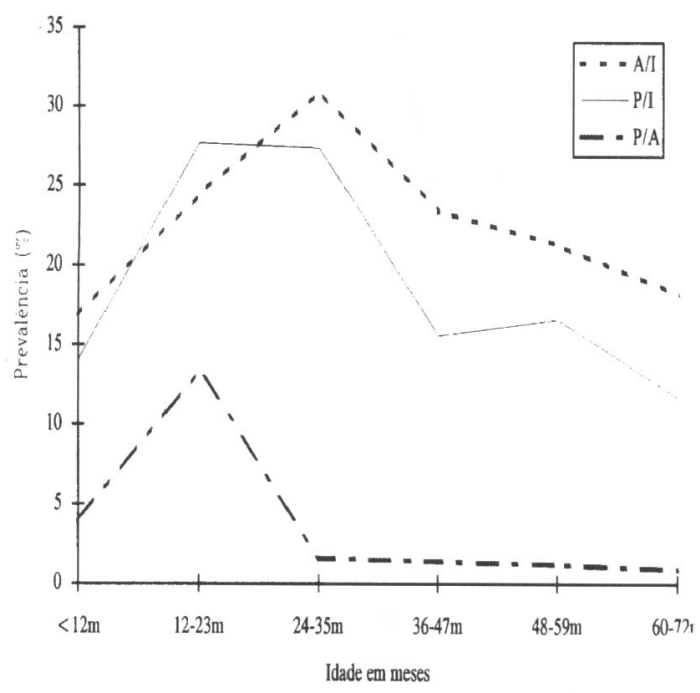

Figura 3 - Variaçăo da prevalência de indicadores antropométricos inadequados ( $<-2,0 \mathrm{DP}$ ) com a idade em pré-escolares no semi-árido da Bahia - 1989.

Não houve evidência de que a distribuição dos três indicadores antropométricos inadequados diferisse segundo o sexo da criança: para $\mathrm{A} / \mathrm{I}$ encontrou-se prevalência de $22,4 \%$ entre os meninos e $23,5 \%$ entre as meninas $(p=0,726)$; em relação a P/I, a prevalência foi de $18,2 \%$ e $20,0 \%$, respectivamente ( $p=0,537)$, e para $P / A$ encontrouse $4,6 \%$ em crianças do sexo masculino e $2,7 \%$ nas do sexo feminino $(\mathrm{p}=0,170)$.

A distribuiçāo dos três indicadores antropométricos abaixo de $-2,0 \mathrm{DP}$, em relação ao nível de escolaridade materna está apresentada na Tabela 3. Nảo se encontrou evidência de que os indicadores altura/idade ou peso/idade inadequados nas crianças diferisse segundo a escolaridade de suas mães ( $p=0,089$ e $p=0,165$, respectivamente). Apesar de não ter sido alcançada significância estatística devese registrar que filhos de mães com escolaridade de segundo grau, quando comparados com filhos de mães analfabetas, mostraram prevalências mais baixas tanto de $\mathrm{A} / \mathrm{I}$ inadequada $(7,7 \%$ contra $25,2 \%$ ) como de P/I $(7,5 \%$ versus $21,6 \%)$; além disto, nenhum caso de P/A abaixo de $-2,0 \mathrm{DP}$ (desnutriçāo atual) foi registrado em familias com māes de educação secundária.

$A$ variação na prevalência de $A / I$ e $P / I$ abaixo de -2,0 DP em relaçāo à renda domiciliar per capital foi altamente significativa $(\mathrm{p}=0,001$ e $p=0,000$, respectivamente) (Tabela 4). A variaçāo na desnutrição atual (P/A) nāo mostrou diferença estatisticamente significante $(p=0,506)$, embora somente um dos 25 casos tenha ocorrido em família com renda igual ou maior que $1 / 2 \mathrm{SM}$ per capita. Para cada nível de renda foi calculada a Razão de Prevalência (RP) e Intervalo de Confiança (IC) tendo-se como referência o grupo de renda mais elevado $(R P=1)$. Encontrou-se que crianças de familias com renda inferior a $1 / 4$ SM per capita tem cerca de duas vezes mais chance de apresentarem altura/idade inadequada $(\mathrm{RP}=2,0$ $\mathrm{IC}=1,2-3,5)$ e peso/idade inadequado ( $\mathrm{RP}=2,2$ $\mathrm{IC}=1,2-4,0)$; as outras razőes de prevalência incluem um dentro de seu intervalo de confiança, não sendo portanto significativas.

\section{Consumo Alimentar}

A adequação do consumo dietético de energia, calculada a partir do inquérito recordatório de $24 \mathrm{~h}$, mostrou que apenas $6,8 \%$ das crianças haviam consumido no dia anterior uma dieta adequada que

Tabela 3 - Distribuiçăo de indicadores antropométricos abaixo de $-2,0$ desvios-padrăo (OP), segundo escolaridade das mâes de pré-escolares do semi-árido da Bahia1989

\begin{tabular}{lccc}
\hline Escolaridade & Altura/lade* & Peso/dade** & Peso/Altura** \\
\hline $\begin{array}{l}\text { Analfabeta } \\
\text { (N=310) }\end{array}$ & $25,2 \%$ & $21,6 \%$ & $4,6 \%$ \\
$\begin{array}{l}\text { Alfabetizada } \\
(\mathrm{N}=124)\end{array}$ & $20,5 \%$ & $18,7 \%$ & $2,5 \%$ \\
$\begin{array}{l}\text { Primária } \\
\text { (N=270) }\end{array}$ & $23,8 \%$ & $17,8 \%$ & $3,8 \%$ \\
$\begin{array}{l}\text { Secundária } \\
(\mathrm{N}=40)\end{array}$ & $7,7 \%$ & $7,5 \%$ & $0,0 \%$ \\
\hline
\end{tabular}


Tabela 4 - Risco de indicadores antroponétricos abaixo de $-2,0$ desvios-padrão (DP), segundo a renda per capita das famílias de pré-escolares do semi-árido da Bahia-1989.

\begin{tabular}{|c|c|c|c|c|c|c|}
\hline \multirow{2}{*}{$\begin{array}{l}\text { Renda familiar } \\
\text { per capita }\end{array}$} & \multicolumn{2}{|c|}{ Altura/ldade' } & \multicolumn{2}{|c|}{ Peso/ldade" } & \multicolumn{2}{|c|}{ Peso/Altura" } \\
\hline & Prevalência & $\mathrm{RP}[\mathrm{IC}]$ & Prevalência & $\mathrm{RP}[\mathrm{IC}]$ & Prevalência & $\mathrm{RP}[\mathrm{IC}]$ \\
\hline $\begin{array}{l}<1 / 4 \mathrm{SM} \\
(\mathrm{N}=367)\end{array}$ & $27,8 \%$ & $2,0[1,2-3,5]$ & $24,5 \%$ & $2,1[1,2-4,0]$ & $3,5 \%$ & $3,5[0,5-150,8]$ \\
\hline $\begin{array}{c}1 / 4 \text { a } 1 / 2 \mathrm{SM} \\
(\mathrm{N}=238)\end{array}$ & $20,2 \%$ & $1,5[0,8-2,6]$ & $15,5 \%$ & $1,4[0,7-2,6]$ & $4,6 \%$ & $4,6[0,6-200,2]$ \\
\hline \multirow[t]{2}{*}{$\begin{array}{r}\geq 1 / 2 \text { SM } \\
(N=96)\end{array}$} & $13,5 \%$ & 1,0 & $11,4 \%$ & 1,0 & $1,0 \%$ & 1,0 \\
\hline & \multicolumn{2}{|c|}{$p=0,001$ (para tendência) } & \multicolumn{2}{|c|}{$“ p=0,000$ (para tendência) } & \multicolumn{2}{|c|}{$\cdots p=0,506$ (para tendencia) } \\
\hline
\end{tabular}

suprisse os requerimentos energéticos para sua faixa etária, e que mais de $30 \%$ delas haviam consumido apenas 50\% dos requerimentos (Tabela 5). Em relação à adequação do consumo de proteína encontrou-se que $79,5 \%$ das crianças consumiram nas últimas $24 \mathrm{~h}$ a recomendação de ingestão diária da FAO/OMS. Os dados relativos ao consumo de ferro e vitamina A foram objeto de outros trabalhos ${ }^{2,18}$.

\section{Discussão e Conclusōes}

A privação nutricional prolongada usualmente acarreta atrasos no desenvolvimento ponderal e no crescimento linear de pré-escolares, os quais são detectados através de indicadores antropométricos; estes têm sido muito empregados como "proxy" para o estado nutricional. Inadequação no indicador peso/altura (P/A) reflete uma perda de peso atual e pode ser interpretado como indicativo de um processo de desnutrição aguda ou atual (emaciação). Privaçð̄es nutricionais por períodos prolongados e/ou sucessivos episódios de perda de peso na infância acabam por acarretar atrasos no crescimento, detectados por inadequação no indicador altura/idade $(A / I)$ o que reflete então uma situação de desnutrição pregressa (nanismo nutri-

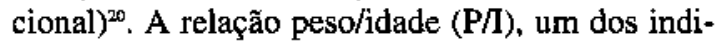
cadores usados com mais frequiência, mostra que

Tabela 5 - Adequação dietética em energia e proteínas de pré-escolares do seml-árido da Bahla - 1989.

\begin{tabular}{lrrrr}
\hline Valor & \multicolumn{2}{c}{ Energia } & \multicolumn{2}{c}{ Proteína } \\
Adequaçăo* & $\mathrm{N}$ & $(\%)$ & \multicolumn{1}{c}{$\mathrm{N}$} & \multicolumn{1}{c}{$(\%)$} \\
\hline $450 \%$ & 186 & 30,2 & 23 & 3,7 \\
$50-74 \%$ & 267 & 43,3 & 34 & 5,5 \\
$75-99 \%$ & 121 & 19,6 & 69 & 11,2 \\
$\geq 100 \%$ & 42 & 6,8 & 490 & 79,5 \\
\hline Total & 616 & 100,0 & 616 & 100,0 \\
\hline
\end{tabular}

- Percentagem da recomendação da FAO/OMS ${ }^{\text {(aes }}$ existe atraso no ganho de peso mas nāo diferencia se o tipo de problema é atual ou pregresso. $O$ padrão mais utilizado para a comparaçāo dos dados antropométricos é o NCHS, apesar do mesmo apresentar algumas limitações técnicas". Além disto, existe o debate sobre sua utilização universal, uma vez que foi derivado de estudos com crianças de países desenvolvidos e de outra composição étnical'. A controvérsia "padrão local" versus "padrāo internacional" persiste, mas atualmente o consenso é que desde o nascimento até a puberdade o padrão de desenvolvimento pondero-estatural é muito mais dependente das condições ambientais (nutrição, saúde e outras) do que de condicionantes genéticos ou étnicos conforme demonstrado por alguns autores ${ }^{10,12}$.

A populaçăo estudada caracterizou-se como tendo sofridos agravos à saúde e nutrição que resultaram em índices antropométricos inadequados. Em relação à altura/idade, que reflete um processo de desnutrição pregressa, a prevalência encontrada na presente pesquisa não diferiu daquela registrada para a populaçāo urbana do Nordeste na pesquisa nacional realizada no mesmo ano; contudo, em relação à desnutriçāo atual (peso/altura), como também para o indicador peso/idade, os pre-escolares do semi-árido apresentaram prevalências significativamente superiores às da populaçāo urbana do Nordeste $(3,6 \%$ versus $2,0 \%$ e $19,1 \%$ versus $10,3 \%$, respectivamente) ${ }^{\text {ls }}$.

A faixa etária em que ocorreu a maior prevalência de índices antropométricos inadequados foi entre os 12 e os 35 meses, fato já encontrado em outros estudos em nosso meio's. A possível inter-relaçāo com o abandono do aleitamento materno e com uma dieta de desmane inadequada, assim como suas implicaçōes programáticas serāo relatados em outro trabalho*.

* Padrdo de consumo alimentar em pré-escolares no semi-árido da Bahia (em preparação). 
Apesar de não haver alcançado significância estatística, a prevalência da desnutriçāo foi cerca de três vezes maior nos filhos de mães analfabetas, comparados com filhos de mães com segundo grau. $O$ padrão de renda das famílias estudadas reflete o baixo poder aquisitivo da população desta região: mais de $2 / 3$ delas recebiam menos que dois salários-mínimos mensais, ponto de corte utilizado com freqüência em programas sociais do governo para definir famílias de baixa renda. Abaixo do nível de renda per capita menor que $1 / 4$ do SM encontravam-se mais da metade das famnilias estudadas.

A renda familiar reflete-se no acesso aos alimentos. Os resultados do ENDEF indicavam que na época daquela pesquisa (1974-75), o consumo de energia era adequado em $57,2 \%$ e o consumo de proteínas em $95,3 \%$ das famílias com crianças de 1 a 5 anos da região urbana do Nordeste 6 . Embora estes dados não possam ser diretamente comparados com os aqui apresentados, uma vez que se referem ao consumo da unidade familiar e não ao da criança, os níveis de inadequação encontrados no presente estudo (consumo adequado de energia em $6,8 \%$ e de proteínas em $79,5 \%$ das crianças) parecem indicar uma deficiência dietética até mais séria do que a da população estudada pelo ENDEF. $O$ inquérito dietético recordatório de $24 \mathrm{~h}$, empregado no presente estudo, tem sido usado extensivamente tanto de forma isolada, como em combinação com outras técnicas de inquérito de consumo alimentar ${ }^{22}$.

\section{Referências Bibliográficas}

1. ASSIS, A.M.O. et al, Expropriação e fome. Salvador, Empresa Gráfica da Bahia, 1987.

2. ASSIS, A.M.O. et al. Anemia pré-escolares no semi-ărido da Bahia. Cadernos de Saúde Pública [no prelo]

3. BAEZ, M. \& MONTEIRO, E.A. Estudo antropométrico da região Nordeste do Brasil. In: Arruda, J.M. et al. Pesquisa nacional sobre saude matemo-infantll e planejamento familiar PNSMIPF - Brasil, 1986. Rio de Janeiro, BEMFAM/IRD, 1987.

4. JOINT FAO/WHO/UNU EXPERT CONSULTATION ON ENERGY AND PROTEIN REQUIREMENTS. Rome, 1981, Report. Geneva, World Health Organization, 1985. (Who-Technical Report Series, 724).

5. FLNDAÇÃO IBGE. Censo demográfico-Babia 1991; resultados preliminares. Rio de Janeiro, 1992. (10\% Recenseamento Geral do Brasil, 1992).

6. FL_DAÇÃO IBGE. Perfil estatístico de crianças e mâes no Brasil: Aspectos nutricionais 1974-
A proporção de desnutridos de acordo com a classificação de Gomez, encontrada população estudada (44,6\% de DI e $12,4 \%$ de DII e DIII cornbinados), é bastante semelhante ao que foi publicado em 3 estudos anteriores no Estado da Bahia, na década de 80 (entre 40,0 e $50,9 \%$ de DI e entre $13,0$ e $19,4 \%$ de DII e DIII combinados) $)^{1,7,8}$.

Nas últimas décadas tem sido demonstrada melhoria significativa nos índices de mortalidade e desnutrição em crianças brasileiras ${ }^{15}$. As prevalências de desnutrição encontradas no presente estudo vêm confirmar o fato de que as condiçōes de vida no semi-árido continuam extremamente difíceis e que as crianças dessa regiāo provavelmente nāo lograram ainda alcançar os mesmos benefícios que o restante da população infantil brasileira.

\section{Agradecimentos}

Às nutricionistas: Maisa C. Martins e Maria da Purificação N. Araújo pela participação na coleta de dados e na análise dos dados de inquérito dietético; Lilian Ramos e Rita Ribeiro pela sua colaboração na coleta e devolução dos dados à comunidade; Teresinha R. S. Raposo pela digitaçāo dos dados; Lilian Cupari, da Escola Paulista de Medicina, pela cessāo do Programa de Apoio a Decisāo em Nutrição; à Prof ${ }^{2}$. Suraiya Ismail, da London School of Hygiene and Tropical Medicine, pelas sugestoes na interpretação do inquérito dietético; às alunas de nutrição que colaboraram na coleta de dados.

5. Rio de Janeiro. 1982

7. FREITAS, M.C.S. \& PRADO, M.S. Expropriaçâo e fome: um estudo de caso em áreas periféricas de Barreiras. In: Assis, A.M.O. et al, Expropriação e fome. Salvador. Empresa Gráfica da Bahia, 1987. p. 112-63.

8. GALDENZI, E. ․ et al. Projeto Cansançâo: uma vivência no Sertào da Babia. Coordenaçào Central de Extensão da LFBA/Imprensa Gráfica da LFBA, Salvador 1992.

9. GOMEZ, F. Desnutrición. Bol Medico Hosp. Infant. Méx., 3: 534-51, 1946.

10. GOPALAN, C. Growth standards for Indian Children. NFI Bull., 10(3): 1-4, 1989.

11. GORSTEIN, J. et al. Issues in the assessment of nutricional status using anthropometry, Bult. World Health Organ., 72: 273-83, 1994.

12. HABICHT, J.P. et al. Height and weight standards for pre-school children: how relevant are ethnic differences in growth potencial? Lancet, 1: 611-5, 1974.

13. JAGLARIBE, H. et al. Reforma ou caos. São Paulo, Paz e Terra. 1689. 
14. MEASURING groups of children. [Editorial], Lancet, 2: 186-7, 1980.

15. MONTEIRO, M.F.G. \& CERVIMI, R. org. Perfil estatístico de crianças $e$ mães no Brasil: aspectos de saúde e nutriçào de crianças no Brasil 1989. Rio de Janeiro, 1992.

16. NATIONAL CENTER FOR HEAITH STATISTICS. Growth curves for children birtb-18 years United States. Washington, D.C., 1977. (DHEW publication 78-1650).

17. PASTORE, J. et al. Mudança social e pobreza no Brastl: 1970-80. Sảo Paulo, FIPE/Pioneira, 1983.

18. SANTOS, L.M.P. et al. Situação nutricional e alimentar de pré-escolares no semi-árido da Bahia (Brasil) II. Hipovitaminose A. Rev.
Satide Pública, 30(1) 1996. (no prelo).

19. SUPERINTENDÊNCIA DE DESENVOLVIMENTO DO NORDESTE (SLDENE). Municipios da região semi-árida do Estado da Bahia (preliminar). SUDENE/DEPG/PSU/SRE. Salvador 1984 (mimeografado)

20. USE and interpretation of anthropometric indicators of nutritional status. Bull. World Health Organ., 64:929-41, 1986.

21. VANUCCHI, H. et al. Nutritive value of a rice and beans based diet for agricultural migrant workers in Southern Brazil. Nutr, Rep. Int. 24: 129-34, 1981

22. WILET, W. Nutritional epidemiology. New York, Oxford University Press, 1990.

\begin{abstract}
A survey of 754 preschool children was undertaken in the urban areas of seven small towns of the semi-arid region of Bahia, Northeast Brazil. The study set out to determine the prevalence of weight and height deficits, as well as to correlate them with variables such as age, family income, mother's schooling and food consumption. A prevalence of $22.9 \%$ of Height for Age (HA) below -2.0 SD was found (stunting) and prevalence of $19.1 \%$ and $3.6 \%$ were determined, respectively for Weight for Age (WA) and Weight for Height (WH) below -2.0 SD (wasting). Regarding food consumption, only $6.8 \%$ of the children received a diet with an adequate energy supply for their age group in the previous day. There was a close association between inadequate HA and WA and family income per capita $(p=0.001$ and $p=0.000$, respectively). Chlidren from families with income < $1 / 4$ of the minimum wage per capita had twice the chance of being mainourished as compared with those from the $\geq 1 / 2$ minimum wage per capita stratum. The children included in this study showed prevalences of inadequate WH and WA significantly higher than those found in a national survey conducted in the same period ( $p=0.047$ and $p=0.000$ respectively). This fact is surprising since in the last decade important reductions in child mainutrition and mortally were reported in the country as a whole; this may indicate that children from this region probably were not benefitted in the same way as the rest of the Brazilian child population.
\end{abstract}

Anemia, epidemiology. Hemoglobins, analysis. 